\title{
Gravitational Wilson loop and large scale curvature
}

\begin{abstract}
H. W. Hamber* and R. M. Williams ${ }^{\dagger}$
Max Planck Institute for Gravitational Physics (Albert Einstein Institute) D-14476 Potsdam Germany

(Received 15 June 2007; published 12 October 2007)

In a quantum theory of gravity the gravitational Wilson loop, defined as a suitable quantum average of a parallel transport operator around a large near-planar loop, provides important information about the large-scale curvature properties of the geometry. Here we shows that such properties can be systematically computed in the strong coupling limit of lattice regularized quantum gravity, by performing local averages over loop bivectors, and over lattice rotations, using an assumed near-uniform measure in group space. We then relate the resulting quantum averages to an expected semiclassical form valid for macroscopic observers, which leads to an identification of the gravitational correlation length appearing in the Wilson loop with an observed large-scale curvature. Our results suggest that strongly coupled gravity leads to a positively curved (de Sitter-like) quantum ground state, implying a positive effective cosmological constant at large distances.
\end{abstract}

DOI: 10.1103/PhysRevD.76.084008

PACS numbers: 04.60. $-\mathrm{m}, 04.60 . \mathrm{Gw}, 04.60 . \mathrm{Nc}$, 98.80.Qc

\section{INTRODUCTION}

An important question for any theory of quantum gravity is what gravitational observables should look like [1], i.e. which expectation values of operators (or ratios thereof) have meaning and physical interpretation in the context of a manifestly covariant formulation, specifically in a situation where metric fluctuations are not necessarily bounded. Such averages naturally include expectation values of the (integrated) scalar curvature and other related quantities (involving, for example, curvature-squared terms), as well as correlations of operators at fixed geodesic distance, sometimes referred to as bilocal operators. Another set of physical observables corresponds to the gravitational analog of the Wilson loop [2], providing information about the parallel transport of vectors, and therefore on the effective curvature, around large, nearplanar loops, and the correlation between particle world lines $[3,4]$ (providing information about the static gravitational potential). In this paper we will concentrate on defining and exploring physical properties of the gravitational Wilson loop [5].

Before embarking on the gravitational case, let us recall more generally the well-known fact that many low-energy physical properties in gauge theories cannot be computed reliably in weak coupling perturbation theory. Thus, for example, in non-Abelian gauge theories a confining potential for static sources placed in the fundamental representation is found at sufficiently strong coupling, by examining the behavior of the Wilson loop [2], defined for a large closed planar loop $C$ as

\footnotetext{
*On leave from the Department of Physics, University of California, Irvine, CA 92717.

${ }^{\dagger}$ Permanent address: Department of Applied Mathematics and Theoretical Physics, Wilberforce Road, Cambridge CB3 0WA, United Kingdom.
}

$$
W(C)=\left\langle\operatorname{tr} \mathcal{P} \exp \left\{i g \oint_{C} A_{\mu}(x) d x^{\mu}\right\}\right\rangle,
$$

with $A_{\mu} \equiv t_{a} A_{\mu}^{a}$ and the $t_{a}$ 's the group generators of $S U(N)$ in the fundamental representation. Specifically, in the pure gauge theory at strong coupling the leading contribution to the Wilson loop can be shown to follow an area law for sufficiently large loops

$$
W(C) \sim_{A \rightarrow \infty} \exp \left(-A(C) / \xi^{2}\right),
$$

where $A(C)$ is the minimal area spanned by the planar loop $C[6,7]$. The quantity $\xi$ is the gauge field correlation length, defined, for example, from the exponential decay of the Euclidean correlation function of two infinitesimal loops separated by a distance $|x|$,

$$
\begin{aligned}
G_{\square}(x)= & \left\langle\operatorname{tr} \mathcal{P} \exp \left\{i g \oint_{C_{\epsilon}^{\prime}} A_{\mu}\left(x^{\prime}\right) d x^{\prime \mu}\right\}\right. \\
& \left.\times(x) \operatorname{tr} \mathcal{P} \exp \left\{i g \oint_{C_{\epsilon}^{\prime \prime}} A_{\mu}\left(x^{\prime \prime}\right) d x^{\prime \prime \mu}\right\}(0)\right\rangle_{c} .
\end{aligned}
$$

Here the $C_{\epsilon}$ 's are two infinitesimal loops centered around $x$ and 0 , respectively, suitably defined on the lattice as elementary square loops, and for which one has at sufficiently large separations

$$
G_{\square}(x) \sim_{|x| \rightarrow \infty} \exp (-|x| / \xi) .
$$

The inverse of the correlation length $\xi$ is known to correspond to the lowest mass excitation in the gauge theory, the scalar glueball.

Not only will we adapt this definition to the gravitational case, but more specifically to the case of discrete gravity, in the context of the discretization scheme known as Regge calculus [8]. It will turn out that it is most easily achieved by using a slight variant of Regge calculus, in which the action coincides with the usual Regge action in the nearflat limit. In Sec. II, we shall describe the lattice notion of 
parallel transport, and how areas are defined on the dual lattice. Then in Sec. III, the gravitational Wilson loop will be defined, and it will be shown why, in the discrete case, we modify the action. In Sec. IV, sample calculations will be performed and the behavior of large Wilson loops derived. Much of what is done will be in close parallel with the procedure in lattice gauge theories (as will be immediately obvious to those familiar with that area). We close with a discussion of the interpretation in semiclassical terms of our main result for a large Wilson loop. We will argue there that our results imply that for strong coupling (large bare Newton's constant $G$ ) the behavior of the Wilson loop is consistent with a positive vacuum curvature, and therefore with (Euclidean) de Sitter space.

\section{ROTATIONS, PARALLEL TRANSPORT, AND VORONOI LOOPS}

In lattice gravity, space-time is built up from flat simplices, with curvature restricted to the subspaces of codimension 2. In four dimensions, this means that the hinges, where the curvature lies, are triangles. The contribution to the action of a hinge is the product of its area, $A_{h}$, with the deficit angle, $\delta_{h}$, there. This is defined to be $2 \pi$ minus the sum of the dihedral angles at that hinge, in the simplices meeting there. We may also define a volume associated with each hinge, $V_{h}$ (see later in this section). Then the lattice action for pure four-dimensional Euclidean gravity with a cosmological constant and the usual Einstein scalar curvature term is

$$
I_{\text {latt }}=\lambda_{0} \sum_{h} V_{h}-k \sum_{h} \delta_{h} A_{h},
$$

with $k=1 /(8 \pi G)$, and $V_{h}, A_{h}$, and $\delta_{h}$ are all functions of the edge lengths, which are the basic variables in the theory, analogous to the metric in the continuum. This action only couples edges which belong either to the same simplex or to a set of neighboring simplices, and can therefore be considered as local, just like the continuum action. It leads to the regularized lattice functional integral [9]

$$
Z_{\text {latt }}=\int\left[d l^{2}\right] \exp \left\{-\lambda_{0} \sum_{h} V_{h}+k \sum_{h} \delta_{h} A_{h}\right\},
$$

where, as customary, the lattice ultraviolet cutoff is set equal to one (i.e. all length scales are measured in units of the lattice cutoff). The lattice partition function $Z_{\text {latt }}$ should then be compared to the continuum Euclidean Feynman path integral

$$
Z_{\mathrm{cont}}=\int\left[d g_{\mu \nu}\right] \exp \left\{-\lambda_{0} \int d x \sqrt{g}+\frac{1}{16 \pi G} \int d x \sqrt{g} R\right\} .
$$

In practice the lattice functional integral $Z_{\text {latt }}$ should be regarded as a regularized form of the continuum Euclidean
Feynman path integral. The latter will involve a functional measure over metrics $g_{\mu \nu}(x)$, usually of the form

$$
\int\left[d g_{\mu \nu}\right] \equiv \prod_{x}[g(x)]^{\sigma / 2} \prod_{\mu \geq \nu} d g_{\mu \nu}(x),
$$

where $\sigma$ is a parameter, constrained by the requirement of a nonsingular measure to $\sigma \geq-(d+1)$. For $\sigma=\frac{1}{2}(d-$ 4) $(d+1)$ one has De Witt's measure, while for $\sigma=$ $-(d+1)$ one recovers the original Misner measure. Here we will mostly be interested in the physical fourdimensional case, for which $d=4$ and therefore $\sigma=0$ in the De Witt measure; the specific form of the functional measure is not expected to play an important role in the following, except that it will assumed to be diffeomorphism invariant [5].

Furthermore, unless stated otherwise, it will be convenient to include the cosmological constant term in the measure as well, since this contribution is ultralocal and contains no derivatives of the metric, giving rise to an effective strong coupling measure $d \mu\left(l^{2}\right)$,

$$
d \mu\left(l^{2}\right) \equiv\left[d l^{2}\right] e^{-\lambda_{0} \sum_{h} V_{h}} .
$$

This last expression represents a fairly nontrivial quantity, both in view of the relative complexity of the expression for the volume of a simplex, and because of the generalized triangle inequality constraints already implicit in the definition of $\left[d l^{2}\right]$.

The main assumption used here regarding this effective strong coupling measure will be the existence of a stable ground state with a well-defined average lattice spacing, as implied by direct numerical evaluations of the lattice integrals in four dimensions, at least for sufficiently strong coupling $[5,10]$. In the following the lattice measure will therefore be assumed to be a suitable discretization of the continuum functional measure, and therefore of the form

$$
\int\left[d l^{2}\right]=\int_{0}^{\infty} \prod_{s}\left(V_{d}(s)\right)^{\sigma} \prod_{i j} d l_{i j}^{2} \Theta\left[l_{i j}^{2}\right],
$$

with $\sigma$ again a real parameter, and $\Theta$ a function of the squared edge lengths, ensuring the validity of the triangle inequalities.

At strong coupling the measure and cosmological constant terms form the dominant part of the functional integral, since the Einstein part of the action is vanishingly small in this limit. Yet, and in contrast to strongly coupled lattice Yang-Mills theories, the functional integral is still nontrivial to compute analytically in this limit, mainly due to the triangle inequality constraints. Therefore, in order to be able to derive some analytical estimates for correlation functions in the strong coupling limit, one needs still to develop some set of approximation methods, which will discussed below. These methods and their results can later be tested by numerical means, for example, by integrating 
directly over edges, through the explicit lattice measure over edges given above.

One approach that appears natural in the gravity context follows along the lines of what is normally done in gauge theories, namely, an integration over compact group variables, using the invariant measure over the gauge group [2]. It is of this method that we wish to take advantage here, as we believe that it is well suited for gravity as well. In order to apply such a technique to gravity one needs (i) to formulate the lattice theory in such a way that group variables are separated and therefore appear explicitly; (ii) integrate over the group variables using an invariant measure; and (iii) approximate the relevant correlation functions in such a way that the group integration can be performed exactly, using, for example, mean-field methods for the parts that appear less tractable. In such a program one is aided, as will be shown below, by the fact that in the strong coupling limit one is expanding about a well-defined ground state, and that the measure and the interactions are local, coupling only lattice variable (edges or rotations) which are a few lattice spacings apart, thus excluding the appearance of long-range (powerlike) correlations.

The downside of such methods is that one is no longer evaluating the functional integral for quantum gravity exactly, even in the strong coupling limit; the upside is that one obtains a clear analytical estimate, which later can be in principle systematically tested by numerical methods (which are exact).

In the gravity case the analogs of the gauge variables of Yang-Mills theories are given by the connection, so it is natural therefore to look for a first order formulation of gravity. In a first order formalism one writes for the Einstein-Hilbert pure gravity Lagrangian density

$$
\mathcal{L}=\frac{1}{16 \pi G} \sqrt{g} g^{\mu \nu} R_{\mu \nu} .
$$

For any space-time manifold with an affine connection one has for the Ricci tensor

$$
R_{\mu \nu}=g^{\lambda \sigma} R_{\lambda \mu \sigma \nu}
$$

where

$$
R_{\mu \nu \sigma}^{\lambda}=\partial_{\nu} \Gamma_{\mu \sigma}^{\lambda}-\partial_{\sigma} \Gamma_{\mu \nu}^{\lambda}+\Gamma_{\mu \sigma}^{\eta} \Gamma_{\nu \eta}^{\lambda}-\Gamma_{\mu \nu}^{\eta} \Gamma_{\sigma \eta}^{\lambda} .
$$

Variation of the pure gravitational action requires that

$$
\frac{1}{16 \pi G} \int d^{4} x \delta\left[\sqrt{g} g^{\mu \nu} R_{\mu \nu}\right]=0 .
$$

The variation of the first and second terms inside the square parentheses are trivial, and the variation of $R_{\mu \nu}$ can be simplified by virtue of the Palatini identity

$$
\delta R^{\lambda}{ }_{\mu \nu \sigma}=\delta \Gamma_{\mu \sigma ; \nu}^{\lambda}-\delta \Gamma_{\mu \nu ; \sigma}^{\lambda} .
$$

After integrating by parts one can then show that the term involving the variation of the connection $\Gamma$ implies

$$
\partial_{\lambda} g_{\beta \gamma}-g_{\gamma \sigma} \Gamma_{\beta \lambda}^{\sigma}-g_{\beta \sigma} \Gamma_{\gamma \lambda}^{\sigma}=0
$$

(normally just written as $g_{\mu \nu ; \lambda}=0$ ), and which can be inverted to give the usual relationship between the connection $\Gamma$ and the metric $g$ in Riemannian geometry, namely,

$$
\Gamma_{\mu \nu}^{\lambda}=\frac{1}{2} g^{\lambda \sigma}\left(\partial_{\mu} g_{\nu \sigma}+\partial_{\nu} g_{\mu \sigma}-\partial_{\sigma} g_{\mu \nu}\right) .
$$

Equating to zero the coefficients of $\delta g_{\mu \nu}$ gives instead the ten components of Einstein's field equations. The (wellknown) conclusion therefore is that in the quantum theory one can safely consider functionally integrating separately over the affine connection and the metric, treated as independent variables, with the correct relationship between metric and connection arising then as a consequence of the dynamics. For the rest of this section we will follow a similar spirit, separating out explicitly in the lattice action the degrees of freedom corresponding to local rotations (the analogs of the $\Gamma$ 's in the continuum), which we will find to be most conveniently described by orthogonal matrices $\mathbf{R}$, implying a choice of preferred coordinate systems within the simplices.

The next step is a discussion of the properties of local rotation matrices in the context of the lattice theory, and how these relate to the lattice gravitational action. Since in Regge calculus the interior of each simplex $s$ is assumed to be flat, one can assign to it a Lorentz frame $\Sigma(s)$. Furthermore, inside $s$ one can define a $d$-component vector $\phi(s)=\left(\phi_{0} \ldots \phi_{d-1}\right)$. Under a Lorentz transformation of $\Sigma(s)$, described by the $d \times d$ matrix $\Lambda(s)$ satisfying the usual relation for Lorentz transformation matrices $\Lambda^{T} \eta \Lambda=\eta$, with $\eta$ the flat metric, the vector $\phi(s)$ will rotate to

$$
\phi^{\prime}(s)=\Lambda(s) \phi(s) .
$$

The base edge vectors $e_{i}^{\mu}=l_{0 i}^{\mu}(s)$ themselves are of course an example of such a vector.

Next, consider two $d$-simplices, individually labeled by $s$ and $s^{\prime}$, sharing a common face $f\left(s, s^{\prime}\right)$ of dimensionality $d-1$ [10]. It will be convenient to label the $d$ edges residing in the common face $f$ by indices $i, j=1 \ldots d$. Within the first simplex $s$ one can then assign a Lorentz frame $\Sigma(s)$, and similarly within the second $s^{\prime}$ one can assign the frame $\Sigma\left(s^{\prime}\right)$. The $\frac{1}{2} d(d-1)$ edge vectors on the common interface $f\left(s, s^{\prime}\right)$ (corresponding physically to the same edges, viewed from two different coordinate systems) are expected to be related to each other by a Lorentz rotation $\mathbf{R}$,

$$
l_{i j}^{\mu}\left(s^{\prime}\right)=R_{\nu}^{\mu}\left(s^{\prime}, s\right) l_{i j}^{\nu}(s) .
$$

Under individual Lorentz rotations in $s$ and $s^{\prime}$ one has of course a corresponding change in $R$, namely, $R \rightarrow$ $\Lambda\left(s^{\prime}\right) R\left(s^{\prime}, s\right) \Lambda(s)$. In the Euclidean $d$-dimensional case $R$ is an orthogonal matrix, an element of the group $S O(d)$.

In the absence of torsion, one can use the matrix $R\left(s^{\prime}, s\right)$ to describe the parallel transport of any vector $\phi$ from 
simplex $s$ to a neighboring simplex $s^{\prime}$,

$$
\phi^{\mu}\left(s^{\prime}\right)=R_{\nu}^{\mu}\left(s^{\prime}, s\right) \phi^{\nu}(s) .
$$

$R$, therefore, describes a lattice version of the connection $[11,12]$. Indeed in the continuum such a rotation would be described by the matrix

$$
R_{\nu}^{\mu}=\left(e^{\Gamma \cdot d x}\right)^{\mu}{ }_{\nu}
$$

with $\Gamma_{\mu \nu}^{\lambda}$ the affine connection. The coordinate increment $d x$ is interpreted as joining the center of $s$ to the center of $s^{\prime}$, thereby intersecting the face $f\left(s, s^{\prime}\right)$. Note that it is possible to choose coordinates so that $R\left(s, s^{\prime}\right)$ is the unit matrix for one pair of simplices, but it will not then be unity for all other pairs.

One can consider a sequence of rotations along an arbitrary path $P\left(s_{1}, \ldots, s_{n+1}\right)$ going through simplices $s_{1} \ldots s_{n+1}$, whose combined rotation matrix is given by

$$
\mathbf{R}(P)=R\left(s_{n+1}, s_{n}\right) \cdots R\left(s_{2}, s_{1}\right)
$$

and which describes the parallel transport of an arbitrary vector from the interior of simplex $s_{1}$ to the interior of simplex $s_{n+1}$,

$$
\phi^{\mu}\left(s_{n+1}\right)=R_{\nu}^{\mu}(P) \phi^{\nu}\left(s_{1}\right) .
$$

If the initial and final simplices $s_{n+1}$ and $s_{1}$ coincide, one obtains a closed path $C\left(s_{1}, \ldots, s_{n}\right)$, for which the associated expectation value can be considered as the gravitational analog of the Wilson loop. Its combined rotation is given by

$$
\mathbf{R}(C)=R\left(s_{1}, s_{n}\right) \cdots R\left(s_{2}, s_{1}\right) .
$$

Under Lorentz transformations within each simplex $s_{i}$ along the path, one has a pairwise cancellation of the $\Lambda\left(s_{i}\right)$ matrices except at the end points, giving in the closed loop case

$$
\mathbf{R}(C) \rightarrow \Lambda\left(s_{1}\right) \mathbf{R}(C) \Lambda^{T}\left(s_{1}\right) .
$$

Clearly the deviation of the matrix $\mathbf{R}(C)$ from unity is a measure of curvature. Also, the trace $\operatorname{tr} \mathbf{R}(C)$ is independent of the choice of Lorentz frames.

Of particular interest is the elementary loop associated with the smallest nontrivial, segmented parallel transport path one can build on the lattice. One such polygonal path in four dimensions is shown in Fig. 1. In general consider a $(d-2)$-dimensional simplex (hinge) $h$, which will be shared by a certain number $m$ of $d$-simplices, sequentially labeled by $s_{1} \ldots s_{m}$, and whose common faces $f\left(s_{1}, s_{2}\right) \ldots f\left(s_{m-1}, s_{m}\right) f\left(s_{m}, s_{1}\right)$ will also contain the hinge $h$. In four dimensions several four-simplices will contain, and therefore encircle, a given triangle (hinge). In three dimensions the path will encircle an edge, while in two dimensions it will encircle a site. Thus for each hinge $h$ there is a unique elementary closed path $C_{h}$ for which one again can define the ordered product

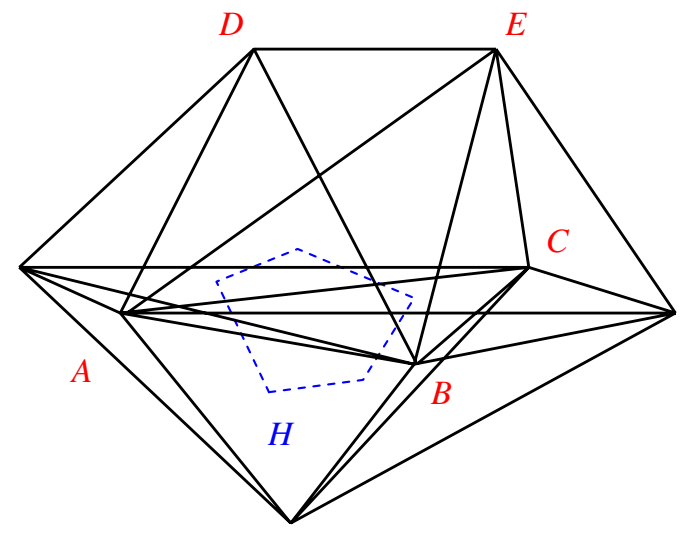

FIG. 1 (color online). Elementary polygonal path around a hinge (triangle) in four dimensions. The hinge $A B C$, contained in the simplex $A B C D E$, is encircled by the polygonal path $H$ connecting the surrounding vertices which reside in the dual lattice. One such vertex is contained within the simplex $A B C D E$.

$$
\mathbf{R}\left(C_{h}\right)=R\left(s_{1}, s_{m}\right) \cdots R\left(s_{2}, s_{1}\right) .
$$

The hinge $h$, being geometrically an object of dimension $(d-2)$, is naturally represented by a tensor of rank $(d-$ 2 ), referred to a coordinate system in $h$ : an edge vector $l_{h}^{\mu}$ in $d=3$, and an area bivector $\frac{1}{2}\left(l_{h}^{\mu} l_{h}^{\prime \nu}-l_{h}^{\nu} l_{h}^{\prime \mu}\right)$ in $d=4$ etc. It will therefore be convenient to define a hinge bivector $U$ in any dimension as

$$
U_{\mu \nu}(h)=\mathcal{N} \epsilon_{\mu \nu \alpha_{1} \alpha_{d-2}} l_{(1)}^{\alpha_{1}} \ldots l_{(d-2)}^{\alpha_{d-2}},
$$

normalized, by the choice of the constant $\mathcal{N}$, in such a way that $U_{\mu \nu} U^{\mu \nu}=2$. In four dimensions

$$
U_{\mu \nu}(h)=\frac{1}{2 A_{h}} \epsilon_{\mu \nu \alpha \beta} l_{1}^{\alpha} l_{2}^{\beta},
$$

where $l_{1}(h)$ and $l_{2}(h)$ two independent edge vectors associated with the hinge $h$, and $A_{h}$ the area of the hinge.

An important aspect related to the rotation of an arbitrary vector, when parallel transported around a hinge $h$, is the fact that, due to the hinge's intrinsic orientation, only components of the vector in the plane perpendicular to the hinge are affected. Since the direction of the hinge $h$ is specified locally by the bivector $U_{\mu \nu}$ of Eq. (28), one can write for the loop rotation matrix $\mathbf{R}$

$$
R_{\nu}^{\mu}(C)=\left(e^{\delta U}\right)_{\nu}^{\mu}
$$

where $C$ is now the small polygonal loop entangling the hinge $h$, and $\delta$ the deficit angle at $h$. One particularly noteworthy aspect of this last result is the fact that the area of the loop $C$ does not enter in the expression for the rotation matrix, only the deficit angle and the hinge direction. Note that in the above expression for the rotation matrix $\mathbf{R}(C)$ both the deficit angle $\delta(C)$ giving the magnitude of the rotation, as well as the bivector $U(C)$ giving the direction of the rotation, are each rather complicated func- 
tions of the original edge lengths, with the latter also depending on a choice for the local coordinate system.

At the same time, in the continuum a vector $V$ carried around an infinitesimal loop of area $A_{C}$ will change by

$$
\Delta V^{\mu}=\frac{1}{2} R_{\nu \lambda \sigma}^{\mu} A^{\lambda \sigma} V^{\nu},
$$

where $A^{\lambda \sigma}$ is an area bivector in the plane of $C$, with squared magnitude $A_{\lambda \sigma} A^{\lambda \sigma}=2 A_{C}^{2}$. Since the change in the vector $V$ is given by $\delta V^{\alpha}=(\mathbf{R}-\mathbf{1})^{\alpha}{ }_{\beta} V^{\beta}$ one is led to the identification

$$
\frac{1}{2} R_{\beta \mu \nu}^{\alpha} A^{\mu \nu}=(\mathbf{R}-\mathbf{1})^{\alpha}{ }_{\beta} .
$$

Consequently the above change in $V$ can equivalently be rewritten in terms of the infinitesimal rotation matrix

$$
R^{\mu}{ }_{\nu}(C)=\left(e^{(1 / 2) R \cdot A}\right)^{\mu}{ }_{\nu}
$$

(where the Riemann tensor appearing in the exponent on the right-hand side should not be confused with the rotation matrix $\mathbf{R}$ on the left-hand side).

The area $A_{C}$ is most suitably defined by introducing the notion of a dual lattice, i.e. a lattice constructed by assigning centers to the simplices, with the polygonal curve $C$ connecting these centers sequentially, and then assigning an area to the interior of this curve. One possible way of assigning such centers is by introducing perpendicular bisectors to the faces of a simplex, and locating the vertices of the dual lattice at their common intersection, a construction originally discussed in $[13,14]$. Another, and perhaps even simpler, possibility is to use a barycentric subdivision. Then the volume element, $V_{h}$, is defined by first joining the vertices of the polyhedron $C$, whose vertices lie in the dual lattice, with the vertices of the hinge $h$, and then computing its volume. We can then show that the polygonal area $A_{C}$ is given by $A_{C}(h)=d V_{h} / V_{h}^{(d-2)}$, where $V_{h}^{(d-2)}$ is the volume of the hinge (a triangle area in four dimensions).

\section{GRAVITATIONAL WILSON LOOP}

We have seen that with each neighboring pair of simplices $s, s+1$ one can associate a Lorentz transformation $R^{\mu}{ }_{\nu}(s, s+1)$, which describes how a given vector $V^{\mu}$ transforms between the local coordinate systems in these two simplices, and that the above transformation is directly related to the continuum path-ordered $(P)$ exponential of the integral of the local affine connection $\Gamma_{\mu \nu}^{\lambda}(x)$ via

$$
R_{\nu}^{\mu}=\left[P e^{\int_{\text {between simplices }}^{\substack{\text { path } \\ \lambda}} d x^{\lambda}}\right]^{\mu}{ }_{\nu},
$$

with the connection having support only on the common interface between the two simplices. Also, for a closed elementary path $C_{h}$ encircling a hinge $h$ and passing through each of the simplices that meet at that hinge, one has for the total rotation matrix $\mathbf{R} \equiv \prod_{s} R_{s, s+1}$ associated with the given hinge

$$
\left[\prod_{s} R_{s, s+1}\right]_{\nu}^{\mu}=\left[e^{\delta(h) U(h)}\right]_{\nu}^{\mu},
$$

as in Eq. (29). This matrix describes the parallel transport of a vector round the loop.

More generally one might want to consider a nearplanar, but noninfinitesimal, closed loop $C$, as shown in Fig. 2. Along this closed loop the overall rotation matrix will still be given by

$$
R_{\nu}^{\mu}(C)=\left[\prod_{s \subset C} R_{S, s+1}\right]_{\nu}^{\mu} .
$$

In analogy with the infinitesimal loop case, one would like to state that for the overall rotation matrix one has

$$
R_{\nu}^{\mu}(C) \approx\left[e^{\delta(C) U(C))}\right]_{\nu}^{\mu},
$$

where $U_{\mu \nu}(C)$ is now an area bivector perpendicular to the loop, which will work only if the loop is close to planar so that $U_{\mu \nu}$ can be taken to be approximately constant along the path $C$. By a near-planar loop around the point $P$, we mean one that is constructed by drawing outgoing geodesics, on a plane through $P$.

If that is true, then one can define an appropriate coordinate scalar by contracting the above rotation matrix $\mathbf{R}(C)$ with the same appropriate bivector, namely,

$$
W(C)=\omega_{\alpha \beta}(C) R^{\alpha \beta}(C),
$$

where the bivector, $\omega_{\alpha \beta}(C)$, is intended as being representative of the overall geometric features of the loop.

In the quantum theory one is of course interested in the average of the above loop operator $W(C)$, as in Eq. (1). The previous construction is indeed quite analogous to the Wilson loop definition in ordinary lattice gauge theories [2], where it is defined via the trace of path-ordered products of $S U(N)$ color rotation matrices. In gravity though

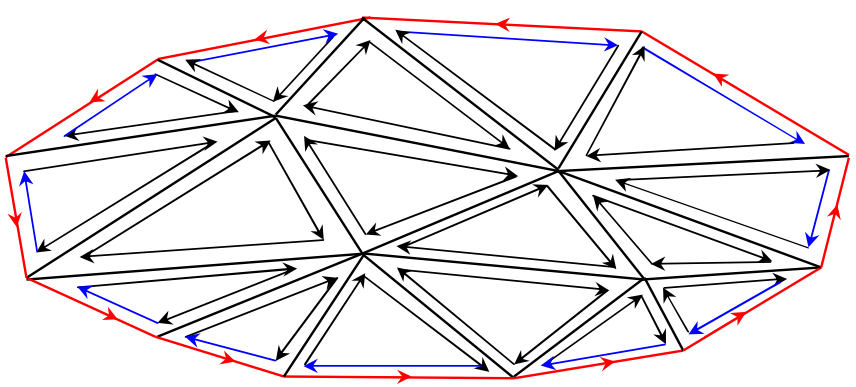

FIG. 2 (color online). Gravitational analog of the Wilson loop. A vector is parallel transported along the larger outer loop. The enclosed minimal surface is tiled with parallel transport polygons, here chosen to be triangles for illustrative purposes. For each link of the dual lattice, the elementary parallel transport matrices $R\left(s, s^{\prime}\right)$ are represented by arrows. In spite of the fact that the (Lorentz) matrices $\mathbf{R}$ can fluctuate strongly in accordance with the local geometry, two contiguous, oppositely oriented arrows always give $R R^{-1}=1$. 
the Wilson loop does not give any information about the static potential $[15,16]$. It seems that the Wilson loop in gravity provides instead some insight into the large-scale curvature of the manifold, just as the infinitesimal loop contribution entering the lattice action of Eqs. (5) and (39) provides, through its averages, insight into the very short distance, local curvature.

Of course for any continuum manifold one can define locally the parallel transport of a vector around a nearplanar loop $C$. Indeed parallel transporting a vector around a closed loop represents a suitable operational way of detecting curvature locally. If the curvature of the manifold is small, one can treat the larger loop the same way as the small one; then the expression of Eq. (36) for the rotation matrix $\mathbf{R}(C)$ associated with a near-planar loop can be rewritten in terms of a surface integral of the large-scale Riemann tensor, projected along the surface area element bivector $A^{\alpha \beta}(C)$ associated with the loop,

$$
R_{\nu}^{\mu}(C) \approx\left[e^{(1 / 2) \int_{S} R{ }_{\alpha \beta} A^{\alpha \beta}(C)}\right]_{\nu}^{\mu} .
$$

Thus a direct calculation of the Wilson loop provides a way of determining the effective curvature at large distance scales, even in the case where short distance fluctuations in the metric may be significant. Conversely, the rotation matrix appearing in the elementary Wilson loop of Eqs. (26) and (29) only provides information about the parallel transport of vectors around infinitesimal loops, with size comparable to the ultraviolet cutoff.

Let us now look in detail at how to construct a Wilson loop in quantum gravity. Since this involves finding the expectation value of a product of rotation matrices round a loop, the natural procedure is to treat these rotation matrices as variables and to integrate over their product, weighted by the exponential of minus the Regge action. The expression for this action has been given in terms of functions of the edge lengths, but an alternative $[11,12,17]$ is to find an expression for it in terms of the rotation matrices. For the dual loop around each hinge, the product of the rotation matrices gives the exponential of the deficit angle, $\delta$, times the rotation generator, $U$ [see Eq. (29)], and we need to find a way of extracting the deficit angle from this product of matrices, at the same time as constructing a scalar function to be averaged. The obvious way of doing this is to contract the product of the $R$-matrices with the rotation generator, $U$, and then take the trace. This is equivalent to the action used in [11,12,17] (see also $[18,19]$ for a hypercubic lattice formulation), obtained by contracting the elementary rotation matrix $\mathbf{R}(C)$ of Eq. (29), with the hinge bivector of Eq. (27),

$$
I_{\text {com }}\left(l^{2}\right)=-\frac{k}{2} \sum_{\text {hinges } \mathrm{h}} A_{h} U_{\alpha \beta}(h) R^{\alpha \beta}(h) .
$$

The above construction can be regarded as analogous to Wilson's lattice gauge theory, for which the action also involves traces of products of $S U(N)$ color rotation matri- ces [2]. This contraction produces the sine of the deficit angle times the area of the triangular hinge and so for small deficit angles it is equivalent to the Regge action. However, in general, away from a situation of small curvatures, the two lattice actions are not equivalent, as can be seen already in two dimensions.

At this stage, we choose to differ from the choice made in [17], for the simple reason that when we come to evaluate Wilson loops, the final result often involves the trace of the bivector $U$, which is zero. Therefore, instead of contracting with $U$, we use a linear combination of it and the unit matrix. In particular, we take the contribution to the action, of a hinge, labeled $h$, to be

$$
I_{h}=\frac{k}{4} A_{h} \operatorname{Tr}\left[\left(U_{h}+\epsilon I_{4}\right)\left(\mathbf{R}_{h}-\mathbf{R}_{h}^{-1}\right)\right],
$$

where $\epsilon$ is an arbitrary multiple of the unit matrix in four dimensions. We have subtracted the inverse of the rotation matrix for the hinge for reasons that will become apparent when we evaluate Wilson loops, and it also plays an important role in the action. At the end, we shall be interested in the limit of small but nonzero $\epsilon$.

The classical action may be evaluated as follows. Since $\mathbf{R}$ equals the exponential of $\delta$ times $U$, it may be expanded in a power series in $\delta$, which is then contracted with the $\left(U+\epsilon I_{4}\right)$ and the trace taken. We use

$$
\operatorname{Tr}\left(U^{2 n+1}\right)=0 ; \quad \operatorname{Tr}\left(U^{2 n}\right)=2(-1)^{n},
$$

to show that

$$
I_{h}=-k A_{h} \sin \left(\delta_{h}\right),
$$

independently of the value of the parameter $\epsilon$. (The unit matrix times the even terms in the power series expansion produces $\operatorname{a} \cos (\delta)$ term, but this cancels between the $\mathbf{R}$ and the $\mathbf{R}^{-1}$ contributions.) Thus $\boldsymbol{\epsilon}$ is in fact an arbitrary parameter, which can be conveniently taken to be nonzero, as we shall see.

There is now a slight amount of freedom in how we define the Wilson loop, for a path $C$ in the dual lattice of a simplicial space. The main choices seem to be

$$
\begin{gathered}
\text { (i) } W(C)=\left\langle\operatorname{Tr}\left(R_{1} R_{2} \ldots R_{n}\right)\right\rangle ; \\
(\text { ii }) \quad W(C)=\left\langle\operatorname{Tr}\left[\left(U_{C}+\epsilon I_{4}\right) R_{1} R_{2} \ldots \ldots R_{n}\right]\right\rangle .
\end{gathered}
$$

Here the $R_{i}$ are the rotation matrices along the path; in (ii), there is a factor of $\left(U_{C}+\epsilon I_{4}\right)$, containing some "average" direction bivector, $U_{C}$, for the loop, which, after all, is assumed to be almost planar. The position of the $U_{C}$ term in the product of $R_{i}$ 's is not arbitrary; to give a unique answer, it needs to be placed before an $R$ which begins one of the plaquette contributions to the action.

We would like to take as independent fluctuating variables the rotation matrices $R_{i}$ and the loop bivectors $U_{i}$, in a first order formalism similar in spirit to that used in [17]. This last statement clearly requires some clarification, as 
both the rotation matrices and the loop bivectors depend on the choice of the original edge lengths, as well as on the orientation of the local coordinate system, and cannot therefore in general be considered as independent variables (as should have already been clear from the detailed discussion of the properties of rotation matrices given in the previous section). On the lattice strong edge length fluctuations get reflected in large fluctuations in the local geometry, which in turn imply large correlated fluctuations in both the deficit angles and in the orientations of the elementary loop. It would therefore seem at first that one would have to integrate over both sets of coupled variables simultaneously, with some nontrivial measure derived from the original lattice measure over edge lengths, which in turn would make the problem of computing the Wilson loop close to intractable, even in the strong coupling limit. In particular one has to take notice of the fact that the lattice deficit angles and the loop bivectors are related to the metric and connection, as they appear in a first order formulation, in a rather nontrivial way.

But there are two important aspects that come into play when evaluating the expectation value of the gravitational Wilson loop for strongly coupled gravity, the first one being that the overall geometric features of the large near-planar loop provide a natural orientation, specified, for example, by a global loop bivector $U_{C}$. As will become clear from explicit calculations given below, in the strong coupling limit the tiling of the large Wilson loop surface by elementary parallel transport loops, which in general have random orientations, requires that their normals be preferentially oriented perpendicular to the plane of the loops, since otherwise a nonminimal surface must result, which leads to a necessarily higher order contribution in the strong coupling limit.

In the case of a hinge surrounded by the large loop with bivector $U_{C}$, one is therefore allowed to write for the bivector operator $U_{h}$ associated with that hinge, labeled by $h$,

$$
U_{h}=U_{C}+\delta U_{h},
$$

where $\delta U_{h}$ is the quantum fluctuation associated with hinge bivector at $h$. But assuming the fluctuation in $\delta U_{h}$ to be zero is an unnecessarily strong requirement, and in the following it will be sufficient to take $\left\langle\delta U_{h}\right\rangle=0$ and $\left\langle\left(\delta U_{h}\right)^{2}\right\rangle \neq 0$, which can be regarded as a mean-field type treatment for the loop bivectors. It will be important therefore in the following to keep in mind this distinction between the fluctutating hinge bivector $U_{h}$, and its quantum average.

The second important aspect of the calculation is that at strong coupling the edge lengths, and therefore the local geometry, fluctuate in a way that is uncorrelated over distances greater than a few lattice spacing. Thus, mainly due to the ultralocal nature of the gravitational lattice measure at strong coupling, the fluctuations in the $U^{\prime} s$ can be taken as essentially uncorrelated as well, again over distances greater than a few lattice spacings, which further simplifies the problem considerably.

One would expect that for a geometry fluctuating strongly at short distances (corresponding therefore to the small $k$ limit) the infinitesimal parallel transport matrices $R\left(s, s^{\prime}\right)$ should be distributed close to randomly, with a measure close to the uniform Haar measure, and with little correlation between neighboring hinges. In such instance one would have for the local quantum averages of the infinitesimal lattice parallel transports $\langle R\rangle=0$, but $\left\langle R R^{-1}\right\rangle \neq 0$, which would require, for a nonvanishing lowest order contribution to the Wilson loop, that the loop at least be tiled by elementary loops with action contributions from Eqs. (5) or (39), thus forming a minimal surface spanning the loop. Then, in close analogy to the YangMills case of Eq. (2) (as a general reference, see for example [20]), the leading contribution to the gravitational Wilson loop would be expected to follow an area law,

$$
W(C) \sim \operatorname{const} k^{A(C)} \sim \exp \left(-A(C) / \xi^{2}\right),
$$

where $A(C)$ is the minimal physical area spanned by the near-planar loop $C$, and $\xi$ the gravitational correlation length, equal to $\xi=1 / \sqrt{|\ln k|}$ for small $k$. For a close-tocircular loop of perimeter $P$ one would use $A(C) \approx P^{2} / 4 \pi$.

We choose now to focus on the Euclidean case in four dimensions, where the rotation matrices will be elements of $S O(4)$. In evaluating the averages over the rotation matrices in the expectation values in the Wilson loops, the integrations we have to perform will be of the form

$$
\begin{aligned}
& \int\left(\prod_{i=1}^{n} d \mu_{H}\left(R_{i}\right)\right) \operatorname{Tr}\left[\ldots\left(U_{j}+\epsilon I_{4}\right) \ldots R_{k} \ldots\right] \\
& \quad \times \exp \left(-\frac{k}{4} \sum_{\text {hingesh }} A_{h} \operatorname{Tr}\left[\left(U_{h}+\epsilon I_{4}\right)\left(\mathbf{R}_{h}-\mathbf{R}_{h}^{-1}\right)\right]\right) / \mathcal{N},
\end{aligned}
$$

where the normalization factor is given by

$$
\begin{aligned}
\mathcal{N}= & \int\left(\prod_{i=1}^{n} d \mu_{H}\left(R_{i}\right)\right) \exp \left(-\frac{k}{4} \sum_{\text {hingesh }} A_{h} \operatorname{Tr}\left[\left(U_{h}+\epsilon I_{4}\right)\right.\right. \\
& \left.\left.\times\left(\mathbf{R}_{h}-\mathbf{R}_{h}^{-1}\right)\right]\right) .
\end{aligned}
$$

This factor will be omitted from subsequent expressions, for notational simplicity.

For smooth enough geometries, with small curvatures, the rotation matrices can be chosen to be close to the identity. Small fluctuations in the geometry will then imply small deviations in the $R$ 's from the identity matrix. However, for strong coupling $(k \rightarrow 0)$ the usual lattice measure $\int d \mu\left(l^{2}\right)$ [21] does not significantly restrict fluctuations in the lattice metric field. As a result we will assume that these fields can be regarded, at least in this regime, as basically unconstrained random variables, only 
subject to the relatively mild constraints implicit in the measure $d \mu\left(l^{2}\right)$. Thus as $k \rightarrow 0$, the geometry is generally far from smooth, since there is no coupling term to enforce long-range order (the coefficient of the lattice Einstein term goes to zero), and one has as a consequence large local fluctuations in the geometry. The matrices $\mathbf{R}$ will therefore fluctuate with the local geometry, and average out to zero, or a value close to zero, in the sense that, for example, the $S O(4)$ rotation

$$
R_{\theta}=\left(\begin{array}{cccc}
\cos \theta & -\sin \theta & 0 & 0 \\
\sin \theta & \cos \theta & 0 & 0 \\
0 & 0 & 1 & 0 \\
0 & 0 & 0 & 1
\end{array}\right)
$$

averages out to zero when integrated over $\theta$. In general an element of $S O(n)$ is described by $n(n-1) / 2$ independent parameters, which in the case at hand can be conveniently chosen as the six $S O(4)$ Euler angles. The uniform (Haar) measure over the group is then

$$
\begin{aligned}
d \mu_{H}(R)= & \frac{1}{32 \pi^{9}} \int_{0}^{2 \pi} d \theta_{1} \int_{0}^{\pi} d \theta_{2} \int_{0}^{\pi} d \theta_{3} \\
& \times \int_{0}^{\pi} d \theta_{4} \sin \theta_{4} \int_{0}^{\pi} d \theta_{5} \sin \theta_{5} \int_{0}^{\pi} d \theta_{6} \sin ^{2} \theta_{6} .
\end{aligned}
$$

This is just a special case of the general $n$ result, which reads

$$
d \mu_{H}(R)=\left(\prod_{i=1}^{n} \Gamma(i / 2) / 2^{n} \pi^{n(n+1) / 2}\right) \prod_{i=1}^{n-1} \prod_{j=1}^{i} \sin ^{j-1} \theta^{i}{ }_{j} d \theta^{i}{ }_{j}
$$

with $0 \leq \theta^{1}{ }_{k}<2 \pi, 0 \leq \theta^{j}{ }_{k}<\pi$ [22].

These averaging properties of rotations are quite similar to what happens in $S U(N)$ Yang-Mills theories, or even more simply in (compact) QED, where the analogs of the $S O(d)$ rotation matrices $\mathbf{R}$ are phase factors $U_{\mu}(x)=$ $e^{i a A_{\mu}(x)}$. There one has the trivial group averaging property $\int \frac{d A_{\mu}}{2 \pi} U_{\mu}(x)=0$ and $\int \frac{d A_{\mu}}{2 \pi} U_{\mu}(x) U_{\mu}^{\dagger}(x)=1$. In addition, for two contiguous closed paths $C_{1}$ and $C_{2}$ sharing a common side one has

$$
e^{i \oint_{C_{1}} \mathbf{A} \cdot \mathbf{d} \mathbf{l}} e^{i \oint_{C_{2}} \mathbf{A} \cdot \mathbf{d} \mathbf{l}}=e^{i \oint_{C} \mathbf{A} \cdot \mathbf{d} \mathbf{l}}=e^{i \int_{S} \mathbf{B} \cdot \mathbf{n} d A},
$$

with $C$ the slightly larger path encircling the two loops. For a closed surface tiled with many contiguous infinitesimal closed loops the last expression evaluates to 1, due to the divergence theorem. In the lattice gravity case the discrete analog of this last result represents the (exact) lattice analog of the contracted Bianchi identities [23].

In practice, luckily, we do not have to explicitly integrate over $S O(4)$ angles, but rather use the following properties of the Haar measure, normalized to one, on the group:

$$
\begin{gathered}
\int d_{H} R=1 ; \\
\int d_{H} R \operatorname{Tr}(A R) \operatorname{Tr}\left(R^{-1} B\right)=\frac{1}{4} \operatorname{Tr}(A B),
\end{gathered}
$$

for arbitrary $4 \times 4$ matrices $A$ and $B$, which also implies

$$
\int d_{H} R R_{i j} R_{k l}^{-1}=\frac{1}{4} \delta_{i l} \delta_{j k} .
$$

As stated previously, we will regard the individual hinge bivectors $U_{h}$ as aligned on average with the Wilson loop bivector $U_{C}$. Alternatively, one can perform the following simple exercise, in which one assumes each hinge bivector $U_{h}$ is aligned in general (arbitrary) directions and performs an integration over those directions. Here we will give an example of such a calculation.

Of course the normals to a 2-d loop in a 4-d space form a plane, rather than a single direction, so in general what one needs to do is an integration over the directions in that plane. However in this example, we are not restricting the plane of the loop, so the integration is a four-dimensional one over all possible directions. It is performed as follows: in the definition of $U_{\alpha \beta}$ in Eq. (28), we put

$$
l_{1}^{\alpha}=\left|l_{1}\right| a^{\alpha}, \quad l_{2}^{\beta}=\left|l_{2}\right| b^{\beta},
$$

with $a_{\gamma} a^{\gamma}=b_{\gamma} b^{\gamma}=1$. Since $A_{h}=\frac{1}{2}\left|l_{1}\right|\left|l_{2}\right| \sin \phi$, where $\phi$ is the angle between the vectors $l_{1}$ and $l_{2}$, the dependence on the magnitudes of those two vectors cancels from the expression for $U$ and we obtain

$$
U_{\alpha \beta}=\frac{\epsilon_{\alpha \beta \gamma \delta} a^{\gamma} b^{\delta}}{\sin \phi},
$$

with $\cos \phi=a_{\gamma} b^{\gamma}$. The integration of $U_{\alpha \beta}$ then becomes

$$
\begin{aligned}
& \frac{4}{\pi^{4}} \prod_{i=1}^{4} \int_{-1}^{1} d a_{i} \prod_{j=1}^{4} \int_{-1}^{1} d b_{j} \\
& \times \frac{\epsilon_{\alpha \beta \gamma \delta} a^{\gamma} b^{\delta} \delta\left(a_{\lambda} a^{\lambda}-1\right) \delta\left(b_{\rho} b^{\rho}-1\right)}{\sqrt{1-\left(a_{\mu} b^{\mu}\right)^{2}}},
\end{aligned}
$$

where the coefficient in front of the integral ensures that the measure is normalized to 1 .

\section{EVALUATION OF WILSON LOOPS AND BEHAVIOR FOR LARGE LOOPS}

In this section, we first evaluate $\langle W\rangle$ for some simple loops and then discuss the general behavior for arbitrary loops, ending with a consideration of the asymptotic behavior for large loops. We shall work out $\langle W\rangle$ for the two possible definitions listed in the previous section. 


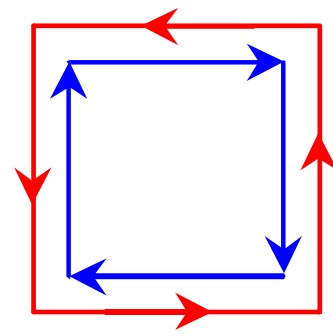

FIG. 3 (color online). A parallel transport loop with four oriented links on the boundary. The parallel transport matrices $\mathbf{R}$ along the links, represented here by arrows, appear in pairs and are sequentially integrated over using the uniform measure.

\section{A. Loop round a single hinge}

Consider a single hinge of area $A$, at which four 4simplices meet (see Fig. 3.). The loop $C$ will consist of four segments between the Voronoi centers of the simplices. Let the rotation matrices on these segments be $R_{1}, R_{2}$, $R_{3}, R_{4}$, and the rotation generator for the hinge $U$. Then we have either

$$
\text { (i) } W(C)=\left\langle\operatorname{Tr}\left(R_{1} R_{2} R_{3} R_{4}\right)\right\rangle,
$$

or

$$
\text { (ii) } W(C)=\left\langle\operatorname{Tr}\left[\left(U+\epsilon I_{4}\right) R_{1} R_{2} R_{3} R_{4}\right]\right\rangle .
$$

Since the only nonvanishing contribution to the integration over the $R$ 's will come from the product of an $R_{i}$ with the corresponding $R_{i}^{-1}$, then the lowest order contribution in $k$ will come from the term in the expansion of the exponential of minus the action which is linear in $\mathbf{R}^{-1}$. Thus in case (i) we obtain

$$
\begin{aligned}
& \frac{k}{4} A \int d_{H} R_{1} d_{H} R_{2} d_{H} R_{3} d_{H} R_{4} \operatorname{Tr}\left(R_{1} R_{2} R_{3} R_{4}\right) \\
& \times \operatorname{Tr}\left[\left(U+\epsilon I_{4}\right) R_{4}^{-1} R_{3}^{-1} R_{2}^{-1} R_{1}^{-1}\right],
\end{aligned}
$$

and a similar expression for (ii), with the extra factor of $\left(U+\epsilon I_{4}\right)$ inserted. Integration over the $R$ 's results in

$$
\text { (i) } \frac{k}{4} \frac{1}{4} A \operatorname{Tr}\left(U+\epsilon I_{4}\right)=\frac{k}{4} A \epsilon \text {, }
$$

or

$$
\text { (ii) } \frac{k}{4} \frac{1}{4} A \operatorname{Tr}\left(U^{2}+\epsilon^{2} I_{4}\right)=-\frac{k}{8} A\left(1-2 \epsilon^{2}\right) \text {, }
$$

and integration over the $U$ 's (a sum over all possible orientations of the loop) is trivial.

\section{B. Loop round two hinges}

Suppose now that the loop goes around two adjacent hinges, with rotation bivectors $U_{1}, U_{2}$. The $R$ matrices going clockwise round the loop in Fig. 4., starting at the top left hand corner, are labeled 1, 2, 5, 6, 7, 4 and the one between the two loops is $R_{3}$.

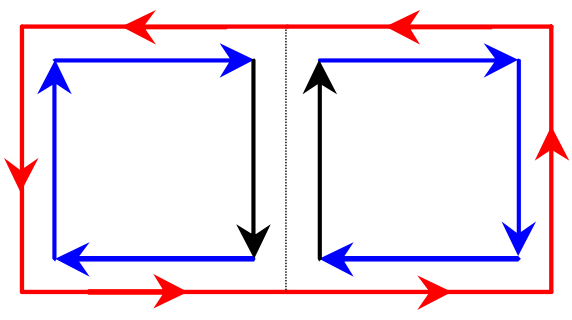

FIG. 4 (color online). A parallel transport loop with six oriented links on the boundary.

Then the possible values of the Wilson loop are

$$
\text { (i) } W(C)=\left\langle\operatorname{Tr}\left(R_{1} R_{2} R_{5} R_{6} R_{7} R_{4}\right)\right\rangle,
$$

or

$$
\text { (ii) } W(C)=\left\langle\operatorname{Tr}\left[\left(U_{C}+\epsilon I_{4}\right) R_{1} R_{2} R_{5} R_{6} R_{7} R_{4}\right]\right\rangle .
$$

This time, the lowest order nonzero contribution will come from the term in the expansion of the exponential which is quadratic and involves the product of the $\mathbf{R}^{-1}$ 's from the two hinges, so for (i) the integration over the $R$ 's gives

$$
\begin{aligned}
& \frac{k^{2}}{16} A_{1} A_{2} \int d_{H} R_{1} d_{H} R_{2} d_{H} R_{3} d_{H} R_{4} d_{H} R_{5} d_{H} R_{6} d_{H} R_{7} \\
& \quad \times \operatorname{Tr}\left(R_{1} R_{2} R_{5} R_{6} R_{7} R_{4}\right) \operatorname{Tr}\left[\left(U_{1}+\epsilon I_{4}\right) R_{4}^{-1} R_{3}^{-1} R_{2}^{-1} R_{1}^{-1}\right] \\
& \quad \times \operatorname{Tr}\left[\left(U_{2}+\epsilon I_{4}\right) R_{3} R_{7}^{-1} R_{6}^{-1} R_{5}^{-1}\right],
\end{aligned}
$$

and a similar expression for (ii). Evaluation of the integrals leads to

(i) $\frac{k^{2}}{16} \frac{1}{4^{3}} A_{1} A_{2} \operatorname{Tr}\left(U_{1}+\epsilon I_{4}\right) \operatorname{Tr}\left(U_{2}+\epsilon I_{4}\right)=\frac{k^{2}}{64} A_{1} A_{2} \epsilon^{2}$,

or

$$
\text { (ii) } \begin{gathered}
\frac{k^{2}}{16} \frac{1}{4^{3}} A_{1} A_{2} \operatorname{Tr}\left[\left(U_{C}+\epsilon I_{4}\right)\left(U_{1}+\epsilon I_{4}\right)\right] \operatorname{Tr}\left(U_{2}+\epsilon I_{4}\right) \\
=\frac{k^{2}}{256} A_{1} A_{2} \epsilon\left[\operatorname{Tr}\left(U_{C} U_{1}\right)+4 \epsilon^{2}\right] .
\end{gathered}
$$

The integration over the $U$ 's, and therefore over the loop's orientation, in Eq. (67) is trivial. In Eq. (68), we either set $U_{1}=U_{C}+\delta U_{1}$, with $\left\langle\delta U_{1}\right\rangle=0$ after which the sum over the loop's orientation also becomes trivial since $\operatorname{Tr}\left(U_{C} U_{1}\right)=-2$, or we can use the following technique to evaluate the integral of $\operatorname{Tr}\left(U_{C} U_{1}\right)$ : we assume (as usual) that $U_{C}$ corresponds to a planar or almost-planar loop and we choose Cartesian coordinates (in the tangent space to it) such that the loop is in the $(1,2)$ plane. We take the generator of $U_{C}$ to be a right-angled triangle in the (3, 4) plane, with $l_{C 1}=(0,0,1,0), l_{C 2}=(0,0,0,1)$, and $A_{C}=1 / 2$. It can then be shown that

$$
\operatorname{Tr}\left(U_{C} U_{1}\right)=-\frac{1}{A}\left[\left(l_{1}\right)_{3}\left(l_{2}\right)_{4}-\left(l_{1}\right)_{4}\left(l_{2}\right)_{3}\right],
$$


where $l_{1}$ and $l_{2}$ are edge vectors for the triangular hinge for $U_{1}$ and $A$ is the triangle's area. The integral to be evaluated here is therefore

$$
\begin{aligned}
-\frac{8}{\pi^{4}} \prod_{i=1}^{4} \int_{-1}^{1} d a_{i} \prod_{j=1}^{4} \int_{-1}^{1} d b_{j} \\
\times \frac{\left(a_{3} b_{4}-a_{4} b_{3}\right) \delta\left(a_{\lambda} a^{\lambda}-1\right) \delta\left(b_{\rho} b^{\rho}-1\right)}{\sqrt{1-\left(a_{\mu} b^{\mu}\right)^{2}}} .
\end{aligned}
$$

It can be shown that this integral is zero (an indication that this might be so is the antisymmetry in $a$ and $b$ in the integrand), and so Eq. (68) becomes identical to Eq. (67), apart from an extra power of $\epsilon$.

\section{Loop with one internal hinge}

We now need to consider the situation where the Wilson loop goes around a number of hinges and there is at least one internal hinge, i.e. a hinge where the elementary loop surrounding it is not part of the Wilson loop. For simplicity, we shall consider the case of one such loop. For the labelling of the rotation matrices and the hinges, the reader can annotate Fig. 5 in a way consistent with the expressions below.

In this case, the lowest order contribution comes from a ninth-order term in the expansion of the exponential of the action. We obtain the following results in the two cases:

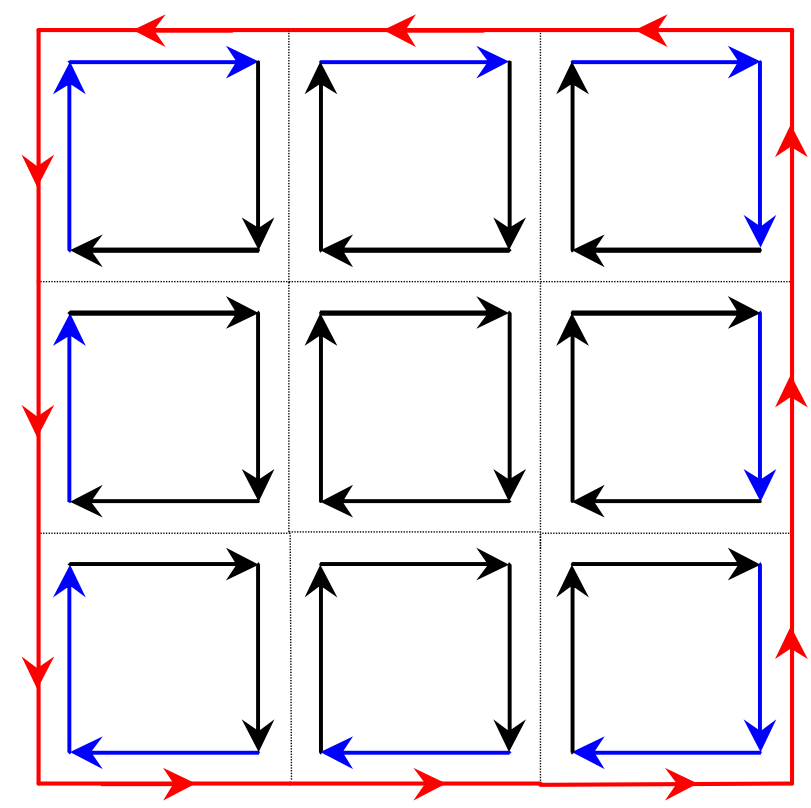

FIG. 5 (color online). A larger parallel transport loop with 12 oriented links on the boundary. As before, the parallel transport matrices along the links appear in pairs and are sequentially integrated over using the uniform measure. The new ingredient in this configuration is an elementary loop at the center not touching the boundary. (i) $\frac{k^{9}}{4^{9}} \frac{1}{4^{17}}\left(\prod_{i=1}^{9} A_{i}\right)\left(\prod_{i=1}^{9} \operatorname{Tr}\left(U_{i}+\epsilon I_{4}\right)\right)=\frac{k^{9}}{4^{17}}\left(\prod_{i=1}^{9} A_{i}\right) \epsilon^{9}$,

or

$$
\begin{aligned}
& \frac{k^{9}}{4^{9}} \frac{1}{4^{17}}\left(\prod_{i=1}^{9} A_{i}\right) \operatorname{Tr}\left[\left(U_{C}+\epsilon I_{4}\right)\left(U_{1}+\epsilon I_{4}\right)\right] \\
& \quad \times\left(\prod_{i=2}^{9} \operatorname{Tr}\left(U_{i}+\epsilon I_{4}\right)\right) \\
& =\frac{k^{9}}{4^{18}}\left(\prod_{i=1}^{9} A_{i}\right) \epsilon^{8}\left[\operatorname{Tr}\left(U_{C} U_{1}\right)+4 \epsilon^{2}\right] .
\end{aligned}
$$

Integration over the $U$ 's is trivial for Eq. (71). In Eq. (68), we either set $U_{1}=U_{C}+\delta U_{1}$, with $\left\langle\delta U_{1}\right\rangle=0$ after which the sum over the loop's orientation also becomes trivial, or we can integrate over the relative orientation of $U_{1}$ relative to $U_{C}$ using the integral formula given at the end of the previous section, after which Eq. (72) gives the same as Eq. (71) but for an extra power of $\epsilon$. The above result also shows that it is better to take $\epsilon>0$, otherwise the answer vanishes to this order. But this is not a problem, as the correct lattice action is recovered irrespective of the value of $\epsilon$, as shown earlier in Eq. (42).

\section{Large loop}

The value of a Wilson loop, in the case when the loop is very large and surrounds $n$ hinges, can be seen to be roughly of the general form

$$
\frac{k^{n}}{4^{2 n}}\left(\prod_{i=1}^{n} A_{i}\right) \epsilon^{\alpha}\left[p+q \epsilon^{2}\right]^{\beta},
$$

where $\alpha+\beta=n$. If $\bar{A}$ is of the order of the geometric or arithmetic mean of the individual loops, this can be approximated by

$$
\left(\frac{k \bar{A}}{16}\right)^{n} \epsilon^{\alpha}\left[p+q \epsilon^{2}\right]^{\beta}
$$

The above result shows again that one should take $\epsilon>0$, otherwise the answer vanishes to this order. As mentioned previously this is quite legitimate, as the correct lattice action is recovered irrespective of the value of $\epsilon$, as in Eq. (42).

Then using $n=A_{C} / \bar{A}$, we may write the area-dependent first factor as

$$
\exp \left[\left(A_{C} / \bar{A}\right) \log (k \bar{A} / 16)\right]=\exp \left(-A_{C} / \xi^{2}\right),
$$

where $\xi=\sqrt{[\bar{A} /|\log (k \bar{A} / 16)|]}$. Recall that this is in the case of strong coupling, when $k \rightarrow 0$. The above is the main result of this paper. The rapid decay of the quantum gravitational Wilson loop as a function of the area is seen here simply as a general and direct consequence of the 
assumed disorder in the uncorrelated fluctuations of the parallel transport matrices $\mathbf{R}\left(s, s^{\prime}\right)$ at strong coupling.

We note here as well that the correlation length $\xi$ is defined independently of the expectation value of the Wilson loop. Indeed a key quantity in gauge theories as well as gravity is the correlation between different plaquettes, which in simplicial gravity is given by

$$
\left\langle(\delta A)_{h}(\delta A)_{h^{\prime}}\right\rangle=\frac{\int d \mu\left(l^{2}\right)(\delta A)_{h}(\delta A)_{h^{\prime}} e^{k \sum_{h} \delta_{h} A_{h}}}{\int d \mu\left(l^{2}\right) e^{k \sum_{h} \delta_{h} A_{h}}} .
$$

In order to achieve a nonvanishing correlation one needs, at least to lowest order, to connect the two hinges by a narrow tube [24], so that

$$
\left\langle(\delta A)_{h}(\delta A)_{h^{\prime}}\right\rangle_{C} \sim\left(k^{n_{t}}\right)^{l} \sim e^{-d\left(h, h^{\prime}\right) / \xi},
$$

where $n_{t} l$ represents the minimal number of dual lattice polygons needed to form a closed surface connecting the hinges $h$ and $h^{\prime}$ (as an example, for a narrow tube made out of cubes connecting two squares one has $\left.n_{t}=4\right)$. In the above expression $d\left(h, h^{\prime}\right)$ represents the actual physical distance between the two hinges, and the correlation length is given in this limit $(k \rightarrow 0)$ by $\xi \sim l_{0} / n_{t}|\log k|$, where $l_{0}$ is the average lattice spacing. Here we have used the usual definition of the correlation length $\xi$, namely, that a generic correlation function is expected to decay as $\exp (-$ distance $/ \xi)$ for large separations. Figure 6 provides an illustration of the situation.

The strong coupling area law behavior predicted for a large Wilson loop in Eq. (75) should be compared with the results for this in numerical simulations of lattice gravity. For small deficit angles (small curvature), the action used in this paper [involving Eq. (40)] is sufficiently close to the usual Regge action of Eq. (5) that the standard simulations can be used for comparison. Universality arguments would suggest a similar behavior for the gravitational Wilson loop for a wide class of lattice actions, constructed so as to reproduce the Einstein-Hilbert continuum action in the continuum limit.

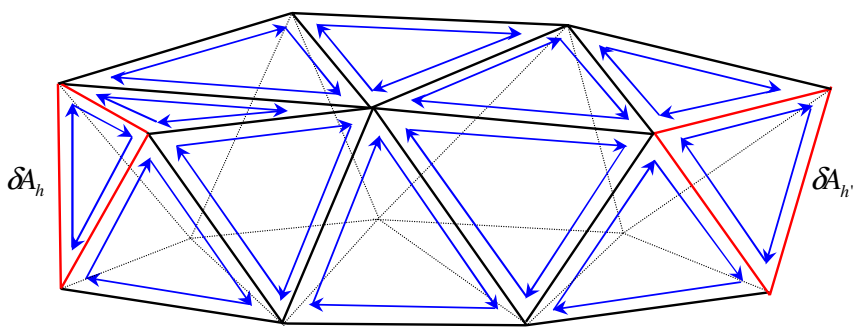

FIG. 6 (color online). Correlations between action contributions on hinge $h$ and hinge $h^{\prime}$ arise to lowest order in the strong coupling expansions from diagrams describing a narrow tube connecting the two hinges. Here vertices represent points in the dual lattice, with the tubelike closed surface tiled with parallel transport polygons. For each link of the dual lattice, the $S O(4)$ parallel transport matrices $\mathbf{R}$ are represented by an arrow.

\section{CONCLUSIONS}

In this paper, we have provided two possible constructions of Wilson loops in the gravitational case and have given explicit calculations for small loops, as well as deriving the asymptotic behavior for large loops. The final step, which we will give here, will be an attempt at providing an interpretation of this last and main result in semiclassical terms. As discussed in the introduction, the rotation matrix appearing in the gravitational Wilson loop can be related classically to a well-defined physical process: a vector is parallel transported around a large loop, and at the end it is compared to its original orientation. The vector's rotation is then directly related to some sort of average curvature enclosed by the loop. The total rotation matrix $\mathbf{R}(C)$ is given in general by a path-ordered $(\mathcal{P})$ exponential of the integral of the affine connection $\Gamma_{\mu \nu}^{\lambda}$ via

$$
R_{\beta}^{\alpha}(C)=\left[\mathcal{P} \exp \left\{\oint_{\text {path C }} \Gamma_{\lambda}^{\cdot} \cdot d x^{\lambda}\right\}\right]_{\beta}^{\alpha} .
$$

In such a semiclassical description of the parallel transport process of a vector around a very large loop, one can reexpress the connection in terms of a suitable coarsegrained, or semiclassical, Riemann tensor, using Stokes' theorem

$$
R^{\alpha}{ }_{\beta}(C) \sim\left[\exp \left\{\frac{1}{2} \int_{S(C)} R_{\cdot \mu \nu} A_{C}^{\mu \nu}\right\}\right]_{\beta}^{\alpha},
$$

where here $A_{C}^{\mu \nu}$ is the usual area bivector associated with the loop in question,

$$
A_{C}^{\mu \nu}=\frac{1}{2} \oint d x^{\mu} x^{\nu}
$$

The use of semiclassical arguments in relating the above rotation matrix $\mathbf{R}(C)$ to the surface integral of the Riemann tensor assumes (as usual in the classical context) that the curvature is slowly varying on the scale of the very large loop. Since the rotation is small for weak curvatures, one can write

$$
R_{\beta}^{\alpha}(C) \sim\left[1+\frac{1}{2} \int_{S(C)} R_{\cdot \mu \nu} A_{C}^{\mu \nu}+\ldots\right]_{\beta}^{\alpha} .
$$

At this stage one is ready to compare the above expression to the quantum result of Eq. (75), and, in particular, one should relate the coefficients of the area terms, which leads to the identification of the magnitude of the large-scale semiclassical curvature with the genuinely quantum quantity $1 / \xi^{2}$. Since one expression [Eq. (81)] is a matrix and the other [Eq. (75)] is a scalar, we shall take the trace after first contracting the rotation matrix with $\left(U_{C}+\epsilon I_{4}\right)$, as in our second definition of the Wilson loop, giving

$$
W(C) \sim \operatorname{Tr}\left(\left(U_{C}+\epsilon I_{4}\right) \exp \left\{\int_{S(C)} R_{\cdot \mu \nu} A_{C}^{\mu \nu}\right\}\right) .
$$

As is standard in simplicial gravity, we use 


$$
R_{\beta \mu \nu}^{\alpha}=\bar{R} U_{\beta}^{\alpha} U_{\mu \nu},
$$

where $\bar{R}$ is some average curvature over the loop, and the $U$ 's here will be taken to coincide with $U_{C}$. The trace of the product of $\left(U_{C}+\epsilon I_{4}\right)$ with this expression gives

$$
\operatorname{Tr}\left(\bar{R} U_{C}^{2} A_{C}\right)=-4 \bar{R} A_{C},
$$

where we have used $U_{\mu \nu} A_{C}^{\mu \nu}=2 A_{C}$ (we choose the directions of the bivectors such that the latter is true for all loops). This is to be compared with the linear term from the other exponential expression, $-A_{C} / \xi^{2}$. Thus the average curvature is computed to be of the order $\bar{R} \sim 1 / \xi^{2}$, at least in the small $k$ limit [25]. An equivalent way of phrasing the last result is that $1 / \xi^{2}$ should be identified, up to a constant of proportionality, with the scaled cosmological constant $\lambda$, which can be regarded as a measure of the intrinsic curvature of the vacuum.

One important aspect of lattice gravity, and of the estimate for the large-scale behavior of the Wilson loop given here, is that it can be tested by numerical methods. That is, the large-scale behavior of the Wilson loop can in principle be computed directly by evaluating the lattice path integral using numerical methods, thus bypassing entirely the need for a separate treatment of the quantum fluctuations in the rotation matrices $\mathbf{R}$ and in the elementary loop bivectors $U$, as was done here in order to obtain an analytical result in the strong coupling limit. Furthermore in a numerical treatment of the Wilson loop one is no longer restricted necessarily to the strong coupling limit.

We see that a direct calculation of the Wilson loop for gravity can provide an insight into whether the manifold is de Sitter or anti-de Sitter at large distances. ${ }^{1}$ Note that the definition of the gravitational Wilson loop is based on a surface with a given boundary $C$, in the simplest case the minimal surface spanning the loop. It is possible though to consider other surfaces built out of elementary parallel transport loops. These will be considered elsewhere.

\section{ACKNOWLEDGMENTS}

The authors wish to thank Hermann Nicolai and the Max Planck Institut für Gravitationsphysik (Albert-EinsteinInstitut) in Potsdam for very warm hospitality. The work of H.W.H. was supported in part by the Max Planck Gesellschaft zur Förderung der Wissenschaften. The work of R.M.W. was supported in part by the UK Particle Physics and Astronomy Research Council.

\footnotetext{
${ }^{1}$ In contrast, numerical studies of full lattice quantum gravity show that the elementary Wilson loop, describing the parallel transport around a single hinge, provides evidence for negative curvature at distance scales comparable to the ultraviolet cutoff [5].
}

[1] S. B. Giddings, D. Marolf, and J. B. Hartle, Phys. Rev. D 74, 064018 (2006).

[2] K. G. Wilson, Phys. Rev. D 10, 2445 (1974).

[3] G. Modanese, Nucl. Phys. B434, 697 (1995).

[4] H. W. Hamber and R. M. Williams, Nucl. Phys. B435, 361 (1995).

[5] H. W. Hamber, Discrete and Continuum Quantum Gravity, AEI report April 2007, and references therein (unpublished).

[6] R. Balian, J. M. Drouffe, and C. Itzykson, Phys. Rev. D 11, 2104 (1975); 19, 2514(E) (1979).

[7] J. M. Drouffe, G. Parisi, and N. Sourlas, Nucl. Phys. B161, 397 (1979).

[8] T. Regge, Nuovo Cimento 19, 558 (1961).

[9] J. B. Hartle, J. Math. Phys. (N.Y.) 26, 804 (1985); 27, 287 (1986); 30, 452 (1989).

[10] H. W. Hamber and R. M. Williams, Nucl. Phys. B248, 392 (1984).

[11] J. Fröhlich, in Non-Perturbative Quantum Field Theory: Mathematical Aspects and Applications, Selected Papers (World Scientific, Singapore, 1992), pp. 523-545.

[12] T.D. Lee, Discrete Mechanics, Proceedings of Erice International School of Subnuclear Physics, 1983, vol. 21 (Plenum Press, New York 1985).

[13] M. G. Voronoi, J. Reine Angew. Math. 134, 198 (1908).

[14] J. L. Meijering, Philips Res. Rep. 8, 270 (1953).
[15] G. Modanese, Phys. Rev. D 47, 502 (1993); 49, 6534 (1994).

[16] H. W. Hamber, Nucl. Phys. B400, 347 (1993).

[17] M. Caselle, A. D’Adda, and L. Magnea, Phys. Lett. B 232, 457 (1989).

[18] S. W. MacDowell and F. Mansouri, Phys. Rev. Lett. 38, 739 (1977); 38, 1376(E) (1977).

[19] C. Mannion and J. G. Taylor, Phys. Lett. 100B, 261 (1981).

[20] M.E. Peskin and D.V. Schroeder, An Introduction to Quantum Field Theory (Addison Wesley, Reading, Massachusetts, 1995), Sec. 22.1.

[21] H.W. Hamber and R. M. Williams, Phys. Rev. D 59, 064014 (1999).

[22] N. Ja. Vilenkin and A. U. Klimyk, Representations of Lie Groups and Special Functions (Kluwer Academic, Dordrecht, Netherlands, 1993), vol. 2, p. 23.

[23] M. Roček and R. M. Williams, in Quantum Structure of Space and Time, edited by M.J. Duff and C. J. Isham (Cambridge University Press, Cambridge, England, 1982), pp. 105-16.

[24] H.W. Hamber and R.M. Williams, Phys. Rev. D 73, 044031 (2006).

[25] H.W. Hamber and R.M. Williams, Phys. Rev. D 75, 084014 (2007). 